\title{
Neurostimulation for treatment-resistant posttraumatic stress disorder: an update on neurocircuitry and therapeutic targets
}

\author{
M. Benjamin Larkin, MD, PharmD, ${ }^{1}$ John P. McGinnis, MD, PhD, ${ }^{1}$ Rita I. Snyder, MD, ${ }^{1}$ \\ Eric A. Storch, PhD, ${ }^{2}$ Wayne K. Goodman, MD, ${ }^{2}$ Ashwin Viswanathan, MD, ${ }^{1}$ and \\ Sameer A. Sheth, MD, PhD'
}

'Department of Neurosurgery and ${ }^{2}$ Menninger Department of Psychiatry and Behavioral Sciences, Baylor College of Medicine, Houston, Texas

\begin{abstract}
Posttraumatic stress disorder (PTSD) is a widespread and often devastating psychiatric condition. Core symptoms include intrusive and distressing thoughts, heightened reactivity, mood changes, cognitive impairments, and consequent avoidance of trauma-related stimuli. Symptoms of PTSD are often refractory to standard treatments, and neuromodulatory techniques have therefore drawn significant interest among the most treatment-resistant patients. Transcranial magnetic stimulation has demonstrated minimal efficacy, and deep brain stimulation trials are currently ongoing. PTSD is a disorder of neural circuitry; the current understanding includes involvement of the amygdala (basolateral and central nuclei), the prefrontal cortex (ventral medial and dorsolateral regions), and the hippocampus. Neuroimaging and optogenetic studies have improved the understanding of large-scale neural networks and the effects of microcircuitry manipulation, respectively. This review discusses the current PTSD literature and ongoing neurostimulation trials, and it highlights the current understanding of neuronal circuit dysfunction in PTSD. The authors emphasize the anatomical correlations of PTSD's hallmark symptoms, offer another potential deep brain stimulation target for PTSD, and note the need for continued research to identify useful biomarkers for the development of closed-loop therapies. Although there is hope that neuromodulation will become a viable treatment modality for PTSD, this concept remains theoretical, and further research should involve institutional review board-approved controlled prospective clinical studies.
\end{abstract}

https://thejns.org/doi/abs/10.3171/2020.4.JNS2061

KEYWORDS closed-loop stimulation; deep brain stimulation; functional neurosurgery; posttraumatic stress disorder; transcranial magnetic stimulation

$\mathrm{P}$ ostTRAumatic stress disorder (PTSD) symptomatology is seen in many historical documents. Nevertheless, this disorder has only recently been formally defined. ${ }^{1}$ PTSD is currently defined as the development of core symptoms after exposure to trauma-either directly experienced, witnessed, or relayed. Hallmarks include intrusive symptoms, persistent avoidance, negative alterations in cognition/mood, and marked alteration in arousal and reactivity $>1$ month, which causes clinically significant social impairment. ${ }^{2}$ There is considerable heterogeneity within the disorder among individuals. In combat-re- lated PTSD, the intrusive and increased arousal symptoms are often more severe. ${ }^{3}$

The prevalence of PTSD is markedly higher among combat veterans-up to $23 \%$ of the 1.64 million veterans returning from Operation Iraqi Freedom/Operation Enduring Freedom will suffer from PTSD. ${ }^{4}$ Furthermore, the financial burden is significant: treatment for all service members in 2005 reached $\$ 1.24$ billion. ${ }^{5}$ Current treatments for PTSD include the use of individual or group forms of cognitive behavioral therapy as well as pharmacotherapy. The efficacy of current US FDA-approved

ABBREVIATIONS ACC = anterior cingulate cortex; BLA = basolateral amygdala; $C E$ = central nuclei; $D B S=$ deep brain stimulation; GABAergic = $y$-aminobutyric acidergic; $\mathrm{mPFC}=$ medial PFC; OFC = orbitofrontal cortex; $\mathrm{PAG}=$ periaqueductal gray; $\mathrm{PCL}=\mathrm{PTSD}$ checklist; $\mathrm{PFC}=$ prefrontal cortex; $\mathrm{PTSD}=$ posttraumatic stress disorder; $\mathrm{RDoC}$ = Research Domain Criteria; TMS = transcranial magnetic stimulation; TR-PTSD = treatment-resistant PTSD; vmPFC = ventral medial PFC. ACCOMPANYING EDITORIAL See pp 1711-1714. DOI: 10.3171/2020.5.JNS201127.

SUBMITTED January 30, 2020. ACCEPTED April 6, 2020.

INCLUDE WHEN CITING Published online July 31, 2020; DOI: 10.3171/2020.4.JNS2061. 
medications has been modest. ${ }^{6,7}$ Currently, accepted psychotherapy strategies include prolonged exposure and cognitive processing therapies. ${ }^{6}$

Combat-related PTSD is more severe and has lower rates of remission than noncombat-related PTSD. ${ }^{8,9}$ There is no consensus on the definition of treatment-resistant PTSD (TR-PTSD), but up to one-third of patients with PTSD fail to attain relief with current evidence-based therapies. Despite this, it is not uncommon for those diagnosed with combat-related PTSD to have spontaneous remission. Commonly used outcome assessment tools for PTSD symptoms include the PTSD Checklist (PCL), Clinician-Administered PTSD Scale, and the Hamilton Rating Scales for Anxiety and Depression. Each of these evaluations uses a series of questions to assess the presence and severity of a patient's PTSD or depression symptoms..$^{10}$ Eftekhari et al. found that 54.5\% of combat veterans had a clinically significant improvement in PCL scores, and $49 \%$ of patients no longer screened positive for PTSD with prolonged exposure therapy. ${ }^{14}$ As many as $39 \%$ of military members will achieve remission by 5 years and up to $55 \%$ by 35 years following trauma. ${ }^{11,12} \mathrm{~A}$ large meta-analysis of 42 studies found that $44 \%$ of victims no longer met criteria for PTSD diagnosis at a mean of 40 months from initial diagnosis using standardized measures. ${ }^{13}$ However, when evaluating results of patient-reported treatment outcomes, it is essential to note the minimal clinically important difference for each assessment tool, because small statistically significant changes may be clinically irrelevant. ${ }^{14}$ Notably, PTSD symptoms are not continuous and are related to internal and/or external triggers. Therefore, a patient may meet remission criteria during one assessment but not another, which suggests the possibility of overestimating remission rates. ${ }^{13}$

Despite improvements, nearly $60 \%$ of individuals still will not achieve remission. Chronic PTSD is linked to decreased life satisfaction, higher rates of depression, and increased suicide risk..$^{15}$ One study found a $17 \%$ mortality rate over a 6-year follow-up period despite treatment. ${ }^{16}$ Thus, novel and effective treatment modalities are needed.

Our understanding of the underlying neurocircuitry must be improved to develop further treatment options for PTSD. There is increasing interest in understanding psychiatric illnesses based on functional domains as opposed to the more categorical typology based on symptom clusters. ${ }^{17}$ The National Institute of Mental Health instituted the Research Domain Criteria (RDoC) project in an effort to advance a multidimensional approach to psychiatric disorders that includes neurobiology and genetics. ${ }^{18}$ This will facilitate objective symptom measurements across functional domains linked to known neural circuits, which may lead to the discovery of new targets and treatment protocols for invasive and noninvasive brain stimulation techniques..$^{19}$ We are unaware of any literature comparing resting-state functional imaging before and after combat deployment, or longitudinal studies of subjects in these populations who ultimately develop PTSD. Future prospective studies identifying these individuals and comparing their functional data to an asymptomatic cohort would also aid in the development of targeted therapies.

Given the need for a comprehensive synthesis of the existing literature, this review discusses previous neuromodulatory and ongoing deep brain stimulation (DBS) trials; highlights the current understanding of the neuronal circuit dysfunction in PTSD and anatomical correlations to symptoms; offers an additional DBS target for PTSD; and emphasizes the need for continued research to identify novel and specific biomarkers for future use in closedloop systems for TR-PTSD.

\section{Methods}

We used PubMed, EMBASE, and ClinicalTrials.gov to query articles for all dates with the major search terms including deep brain stimulation (DBS), transcranial magnetic stimulation (TMS), and posttraumatic stress disorder (PTSD). The following criteria were used for article selection: 1) articles in English, 2) human trials, 3) controlled trials, 4) blinded trials, and 5) randomized trials. In total, 605 articles and 2 clinical trials were identified. After title, abstract, and full-text review, the demographics and results from 7 clinical studies and 2 ongoing clinical trials are presented. This search also revealed a dearth of aggregated information in the literature encompassing pathophysiology, neurocircuitry, and neuromodulation strategies, confirming the existence of an information gap that this review is meant to fill.

\section{The Pathophysiology of PTSD}

\section{Current Model}

PTSD is a complex psychiatric disorder with multiple clinical characteristics; consequently, developing relevant preclinical models has been challenging. ${ }^{20}$ Animal models are imperfect at best, and current PTSD models are adapted only to investigate the short-term outcomes regarding fear and anxiety in response to trauma..$^{21}$ These models translate poorly to the human clinical experience, in which only a small percentage of those who develop an acute response following trauma will develop PTSD. ${ }^{22}$ These models typically evaluate fear conditioning and extinction by pairing a neutral conditioned stimulus with an aversive unconditioned stimulus-e.g., an electrical shock-which results in a new fear response to the previously neutral conditioned stimulus. ${ }^{23}$ The extinction of this response comes from gradual exposure to the conditioned stimulus in the absence of the unconditioned stimulus and the creation of new memories that suppress the initial fear response. Thus, the extinction of the response is the ability to learn that a particular external stimulus no longer needs to be feared. ${ }^{10}$ This process is believed to involve the creation of a new competing memory rather than the erasure of an old one. However, the ability to retain this new memory is thought to be diminished. ${ }^{24}$

\section{PTSD Symptoms and Circuitry}

The symptoms of PTSD are thought to result from reduced top-down control on neuronal circuits, including the amygdala, prefrontal cortex (PFC), and hippocampus, leading to dysfunctional memory processing that continually triggers an overgeneralized fear response. ${ }^{25}$ It is the failure of fear extinction in environments that are nonthreatening but reminiscent of the trauma, as well as a 

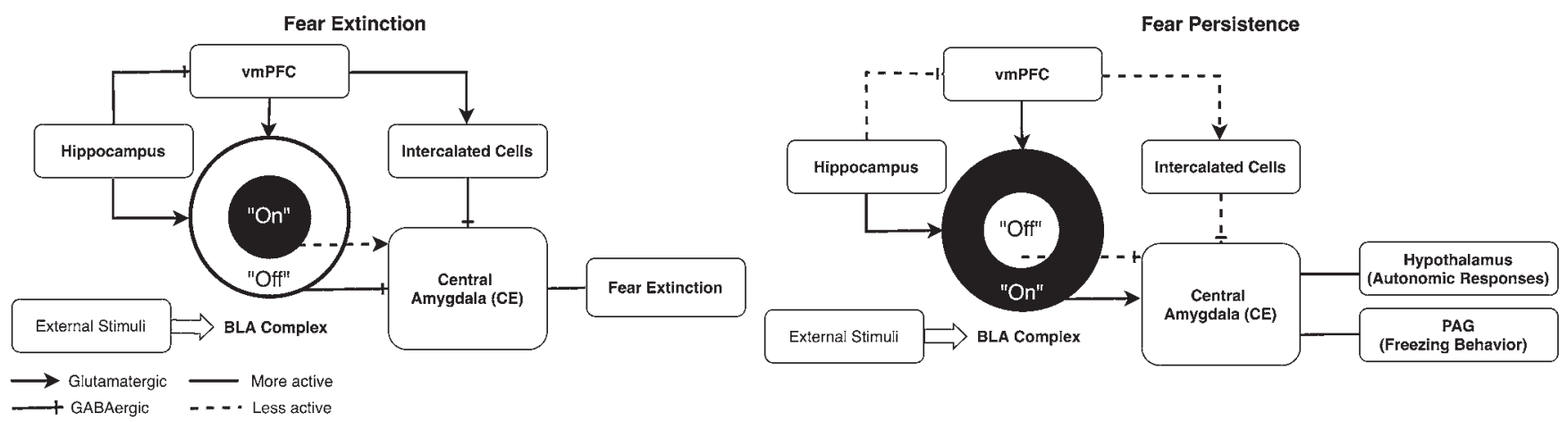

FIG. 1. Diagram of the putative neurocircuitry and pathophysiology of PTSD. In fear extinction, the experience of the external conditioned stimulus is ultimately dissociated from the unconditioned stimulus. The hippocampus and vmPFC play essential roles in memory discrimination and fear extinction. Their interaction with the BLA complex leads to the activation of either predominately "fear on" cells, leading to CE activation and fear persistence, or predominately "fear off" cells, leading to CE inhibition and fear extinction. The vmPFC prevents CE cell activation indirectly via dense glutamatergic connections to intercalated cell neurons. In cases of fear persistence, the CE prompts activation in the hypothalamus and PAG, leading to autonomic and behavioral responses.

generalization to other triggers, that underlies the current understanding of PTSD pathophysiology. ${ }^{10}$

Neuroimaging has advanced our understanding of the neurocircuitry and areas of involvement in PTSD; however, current modalities lack the spatial and temporal resolution necessary to delineate therapeutic targets better. ${ }^{26}$ The expanded use of optogenetics in animal models has further advanced the understanding of microcircuits in PTSD; however, the potential clinical application remains unclear and largely theoretical.

Amygdala. The amygdala is composed of distinct nuclei involved in fear conditioning, including the central nuclei (CE), medial nuclei, intercalated cell clusters, and the basolateral amygdala (BLA) complex. ${ }^{27}$ The BLA complex is composed of mostly (approximately 80\%) glutamatergic spiny neurons, which are tonically inhibited by the remaining $\gamma$-aminobutyric acidergic (GABAergic) interneurons. Fear extinction is thought to be associated with an overall increase in GABAergic tone. ${ }^{28}$ Selective optogenetic activation has helped identify 2 distinct groups of neurons within the amygdala: "fear on" cells, which cause activation of neurons within the CE, and "fear off" cells, which produce $\mathrm{CE}$ inhibition. ${ }^{10}$ The BLA receives numerous projections from cortical and subcortical regions, including the PFC and hippocampus, and it is responsible for the integration and dissemination of stimuli to the CE. The CE, which contains only GABAergic neurons, then modulates responses from the hypothalamus and brainstem regions, such as the periaqueductal gray (PAG), leading to the physiological (autonomic responses), behavioral (freezing), and the overgeneralization of fear conditioning (Fig. 1) ${ }^{20}$ Current twin studies suggest that hyperactivity of the amygdala and dorsal anterior cingulate cortex (ACC) predisposes individuals to the development of PTSD. ${ }^{29,30}$

There are additional hypotheses that regard the amygdala as being intimately involved in behavioral engagement, which implies its involvement with the avoidance symptoms of PTSD. Avoidance behaviors have also been associated with decreased activity in the rostral ACC and right inferior frontal cortex, as well as hyperactivity in the right superior temporal cortex. ${ }^{26}$ Avoidance symptoms do not allow for the creation of new memories that compete with the fear-evoking triggers and are central to the impaired fear extinction in PTSD, which is the basis of cognitive and behavioral therapy approaches in PTSD treatment.

The symptoms of altered arousal and reactivity are generally attributed to hypoactivity of the medial PFC (mPFC) and hippocampus, as well as hyperactivity of the amygdala and bed nucleus of the stria terminalis. ${ }^{31}$ Also, individuals with PTSD commonly exhibit aggression to a perceived threat. The neural circuits regulating aggression are not yet fully understood. However, previous studies suggest the involvement of the amygdala, PAG, and locus ceruleus, which is associated with threat detection and with the autonomic response and altered activity in those with PTSD. ${ }^{32,33}$ Other studies demonstrate aggression and impulsive behavior being related to decreased cortical regulation from $\mathrm{mPFC}$ structures, ventral medial PFC (vmPFC) and dorsal ACC, which recognize threats and determine the appropriate response-as well as the orbitofrontal cortex (OFC) and BLA, which are essential in the regulation and maintenance of the response. ${ }^{34,35}$

Prefrontal Cortex. The intrusive symptoms of PTSD are thought to be due to emotional undermodulation (i.e., a failure of cortical inhibition of the limbic system). ${ }^{36}$ The amygdala is a critical limbic structure associated with emotional reactivity whose inhibition is controlled by the mPFC. ${ }^{37}$ The mPFC plays a crucial role in regulating conditioned fear. ${ }^{28}$ The purpose of the vmPFC is to integrate sensory, emotional, environmental, and memory cues, whereas the dorsolateral PFC regulates appropriate behavioral responses. ${ }^{38}$ Individuals with PTSD demonstrate hypoactivity of the mPFC and a hyperactive amygdala, whereas the extent of fear extinction is associated with the size and activity of vmPFC and amygdala connectivity. ${ }^{39,40}$ Neuroimaging studies of combat veterans demonstrate decreased blood flow in the vmPFC during exposure to 
trauma-related stimuli. ${ }^{39}$ Additionally, those with PTSD show increased insular activity and decreased rostral ACC activity. ${ }^{41}$ The insula is thought to have a role in the sensation of interoception and bodily awareness, whereas the rostral ACC regulates attention, emotion, and arousal. ${ }^{42,43}$

The persistent negative emotional experience found in individuals with PTSD is frequently described as a problem of emotional regulation. ${ }^{26}$ When presented with negative valence imagery, patients with PTSD have difficulty downregulating emotional reactions, such as sadness and anxiety, compared to those without the disorder. ${ }^{44}$ The constricted affect and decreased interest in previously meaningful activities are thought to be related to a dysfunctional reward processing circuitry that involves many areas, including the ventral tegmental area, amygdala, OFC, insula, ACC, dorsomedial PFC, striatum, and the motor cortex. ${ }^{45}$ These areas are involved in seeking rewarding stimuli as well as experiencing pleasure once such rewards are attained. ${ }^{46}$ Hypoactivity of both the striatum and PFC in response to rewarding stimuli has been shown in people who have PTSD. Decreased activity of the vmPFC, anterior insula, and inferior frontal gyrus is also seen in individuals unable to recognize or name emotional states during situations designed to induce symptoms of PTSD. ${ }^{26,47}$ The activity of the mPFC plays a key role in both reward and depression-like behavior; highfrequency burst stimulation of the $\mathrm{mPFC}$ has been shown to exert antidepressant effects. ${ }^{48}$

Individuals with PTSD also demonstrate increased PFC activity during sustained attention and decreased activity with more difficult inhibition tasks, which may reflect a reduced ability to regulate $\mathrm{PFC}$ function during more demanding tasks. ${ }^{49}$ In rodent models the correlation of hippocampus theta oscillations with both theta and single-unit activity in the PFC is important in tests of spatial working memory. Additionally, optogenetic manipulation of ventral hippocampal-PFC afferent pathways was demonstrated to be critical for spatial cues. These studies suggest that dysfunction in these large-scale neural networks affects memory processing that may be crucial in patients with PTSD. ${ }^{26}$

Hippocampus. The hippocampus is crucial for declarative memory and learning processes. Neuroimaging performed to investigate activity of the hippocampus in PTSD patients has been less revealing. ${ }^{20}$ Individuals with PTSD have been shown to have a reduction in hippocampal size; however, it is unclear whether the trauma induces hippocampal atrophy or whether reduced hippocampal volume predisposes someone to developing PTSD. ${ }^{50}$ Studies suggest that pretrauma deficits in executive functioning tasks are further exacerbated following trauma and contribute to a worsened recovery and increased risk of developing PTSD. ${ }^{51}$ However, in rodent models following trauma, acute and chronic corticosteroid secretion induced significant molecular and gene expression changes, resulting in impaired neurogenesis in the dentate gyrus, decreased long-term potentiation in the $\mathrm{CA} 1$, and dendritic retraction in the CA3 regions of the hippocampus. ${ }^{26}$ Additionally, human twin studies suggest that reduced connectivity between the mPFC and hippocampus is acquired and reduces fear extinction capacity. ${ }^{29,30}$

\section{Neuromodulation: Current Status and Future Directions}

The neural circuitry for PTSD is complex, composed of several parallel and inhibitory connections involving both macro- and microcircuits that involve multiple regions of the brain, including the amygdala, PFC, cingulate cortex, insula, and hippocampus, which are implicated in a heterogeneous symptom presentation. Current advances in the understanding of these networks have challenged previous concepts for the pathogenesis of PTSD. It is now understood that PTSD-related dysfunction of various areas results in multiple symptoms of the disorder. ${ }^{26}$ For example, dysfunction of the amygdala can lead to symptoms of intrusion, avoidance, arousal, and reactivity. Likewise, dysfunction of the subdivisions of the PFC results in symptoms of intrusion, as well as negative alterations in cognition and mood. Current advancements in the understanding of the neural circuitry for PTSD have prompted both noninvasive and invasive brain stimulation trialsTMS and DBS, respectively.

Transcranial Magnetic Stimulation. TMS is capable of stimulating brain tissue noninvasively without the surgical risks associated with DBS, albeit with a small risk of seizure induction. Excitation of the cortex occurs with highfrequency stimulation $(>1 \mathrm{~Hz})$, whereas low-frequency stimulation $(\leq 1 \mathrm{~Hz})$ reduces cortical activity. ${ }^{52}$ TMS coils are designed to modulate either small and superficial or broad and deep areas..$^{53}$ The time-varying magnetic field produced by the devices induces an electrical field, the effect of which is governed by the intensity, frequency, and temporal spacing of the delivered electromagnetic energy. ${ }^{54}$

Electroencephalographic studies have shown alpha power decreases in the right hemisphere in patients with PTSD when exposed to trauma-related imagery, which has been further confirmed with the use of SPECT studies with auditory stimulation. ${ }^{55}$ Thus, clinical studies have been aimed at the modulation of the right dorsolateral PFC. Seeking to delineate the benefits of various TMS parameters, multiple studies have suggested some possible clinical benefits compared to placebo, albeit with considerable variability and, in most cases, small sample sizes (Table 1). These studies demonstrate rather small clinical improvement despite significant results, and most studies show notable improvement in sham groups as well. The improvements seen in the sham groups underline the importance of the need for rigorous controls and proper statistical comparisons between intervention and control groups.

Moreover, given the number of scales and time intervals tested in the typical TMS trial, some positive results should be expected by chance alone, even if there were no true effect. Current evidence limits the ability to draw significant conclusions regarding outcomes or optimal treatment parameters. More rigorous research is needed to optimize TMS treatment variables, including the appropriate apparatus, target, stimulation frequency, and parameters, to delineate its true efficacy better.

Deep Brain Stimulation. DBS uses surgically implanted electrodes to deliver electrical stimulation to specific brain targets. The risks of DBS are not insignificant, although the procedure is generally considered to be safe. 
TABLE 1. TMS trials

\begin{tabular}{|c|c|c|c|c|c|c|c|}
\hline $\begin{array}{l}\text { Authors } \\
\text { \& Year }\end{array}$ & $\begin{array}{l}\text { No. } \\
\text { (total) }\end{array}$ & $\begin{array}{l}\text { Outcome } \\
\text { Measures }\end{array}$ & Intensity & $\begin{array}{l}\text { Anatomic } \\
\text { Target }\end{array}$ & Group & $\begin{array}{l}\text { Pulse Frequency/No. of } \\
\text { Pulses/No. of Sessions }\end{array}$ & Results \\
\hline \multirow{2}{*}{$\begin{array}{l}\text { Ahmadizadeh } \\
\text { \& Rezaei, } \\
2018^{67}\end{array}$} & \multirow[t]{2}{*}{58} & \multirow[t]{2}{*}{ PCL-M } & \multirow[t]{2}{*}{$100 \%$} & \multirow[t]{2}{*}{ DLPFC } & Unilat & $20 \mathrm{~Hz} / 1200$ pulses/10 sessions & \multirow{2}{*}{$\begin{array}{l}\text { Significant improvement in both uni- \& } \\
\text { bilat TMS PCL-M score, no signifi- } \\
\text { cant difference btwn groups }\end{array}$} \\
\hline & & & & & Bilat & $20 \mathrm{~Hz} / 1200$ pulses/10 sessions & \\
\hline \multirow{2}{*}{$\begin{array}{l}\text { Cohen et al., } \\
2004^{68}\end{array}$} & \multirow[t]{2}{*}{24} & \multirow{2}{*}{$\begin{array}{l}\text { CAPS, PCL, } \\
\text { TOPTSDS, HAM-A, } \\
\text { HAM-D }\end{array}$} & \multirow[t]{2}{*}{$80 \%$} & \multirow[t]{2}{*}{ rDLPFC } & Low & $1 \mathrm{~Hz} / 100$ pulses/10 sessions & \multirow{2}{*}{$\begin{array}{l}\text { High frequency significantly improved } \\
\text { PCL, TOPTSDS, HAM-A compared } \\
\text { to low frequency \& sham }\end{array}$} \\
\hline & & & & & High & $10 \mathrm{~Hz} / 400$ pulses/10 sessions & \\
\hline $\begin{array}{l}\text { Osuch et al., } \\
2009^{74}\end{array}$ & 9 & $\begin{array}{l}\text { CAPS, SADS, HAM-D, } \\
\text { IES }\end{array}$ & $100 \%$ & rDLPFC & Active & $1 \mathrm{~Hz} / 30$ pulses/20 sessions & No significant difference \\
\hline $\begin{array}{l}\text { Watts et al., } \\
2012^{75}\end{array}$ & 20 & $\begin{array}{l}\text { PCL, BDI, STAI, } \\
\text { BNCE }\end{array}$ & $90 \%$ & rDLPFC & Active & $1 \mathrm{~Hz} / 20$ pulses/10 sessions & $\begin{array}{l}\text { Significant improvement in CAPS, } \\
\text { PCL, \& BDI }\end{array}$ \\
\hline $\begin{array}{l}\text { Isserles et al., } \\
2013^{69}\end{array}$ & 30 & $\begin{array}{l}\text { CAPS, PSS-SR, } \\
\text { HAM-D, BDI }\end{array}$ & $120 \%$ & $\begin{array}{l}\text { Bilat } \\
\text { mPFC }\end{array}$ & Active & $20 \mathrm{~Hz} / 1680$ pulses/12 sessions & $\begin{array}{l}\text { Significant improvement in CAPS for } \\
\text { exposure + stimulation only }\end{array}$ \\
\hline Kozel, $2018^{70}$ & 103 & $\begin{array}{l}\text { CAPS, PCL, M-PTSD, } \\
\text { QIDS, IPF }\end{array}$ & $110 \%$ & rDLPFC & Active & $1 \mathrm{~Hz} / 1800$ pulses/12 sessions & $\begin{array}{l}\text { Significant improvement in CAPS } \\
\text { \& PCL, but more so w/ cognitive } \\
\text { processing therapy }\end{array}$ \\
\hline \multirow{2}{*}{$\begin{array}{l}\text { Kozel et al., } \\
2019^{71}\end{array}$} & \multirow[t]{2}{*}{35} & \multirow{2}{*}{$\begin{array}{l}\text { CAPS, IPF, MADRS, } \\
\text { PCL-5, QIDS-SR, } \\
\text { IPF }\end{array}$} & \multirow[t]{2}{*}{$110 \%$} & \multirow[t]{2}{*}{ rDLPFC } & Low & $1 \mathrm{~Hz} / 2400$ pulses/36 sessions & \multirow{2}{*}{$\begin{array}{l}\text { Significant improvement in CAPS \& } \\
\text { PCL, but no difference btwn low- \& } \\
\text { high-frequency stimulation }\end{array}$} \\
\hline & & & & & High & $10 \mathrm{~Hz} / 2400$ pulses/36 sessions & \\
\hline
\end{tabular}

$\mathrm{BDI}=$ Beck Depression Inventory; BNCE = Brief Neurobehavioral Cognitive Examination; CAPS = Clinician-Administered PTSD Scale; DLPFC $=$ dorsolateral prefrontal cortex; HAM-A = Hamilton Anxiety Rating Scale; HAM-D = Hamilton Depression Rating Scale; IES = Impact of Event Scale; IPF = Inventory of Psychosocial Functioning; M-PTSD = Mississippi Scale for Combat-Related PTSD; MADRS = Montgomery-Åsberg Depression Rating Scale; PCL-M = PTSD Checklist-Military Version; PCL-5 = PTSD Checklist for DSM-5; PSS-SR = PTSD Symptom Scale-Self-Report Version; QIDS = Quick Inventory of Depressive Symptomatology; QIDS-SR = QIDS-Self-Report; rDLPFC = rostral DLPFC; SADS = Schedule for Affective Disorders and Schizophrenia; STAI = State-Trait Anxiety Inventory; TOPTSDS = Treatment Outcome PTSD Scale.

The risk of hemorrhage is approximately $1 \%-3 \%$ per lead, and for infection it is $1 \%-9 \% .{ }^{56,57}$ Although more invasive than TMS, DBS offers the advantage of targeting deeper structures and greater control over how the energy is delivered. However, DBS is limited as well by its broad nature of stimulation to surrounding anatomical structures. As with TMS, the effects of DBS are parameter dependent. It is generally thought that the pulse width determines the size of the region affected by stimulation; that amplitude is related to the strength of the effect; and that frequency determines the network's response-i.e., activation or inhibition. High-frequency stimulation, the most common delivery mode for DBS, has been described as mimicking the effects of a lesion; however, even that common assumption has been challenged. ${ }^{6,58}$

Two ongoing trials are investigating the use of DBS for PTSD: one of the subgenual cingulate and the other targeting the basolateral nucleus of the amygdala (Table 2). There are no currently available published trial data. However, results for 2 patients in the BLA study have been published.$^{59}$ In the first patient there was a $\geq 40 \%$ reduction in symptom severity based on the Clinician-Administered PTSD Scale at 4 years, and resolution of severe combat nightmares and dissociative symptoms after implantation of bilateral ventral BLA leads with high-frequency stimulation (right: $4.2 \mathrm{~V}, 130 \mathrm{~Hz}, 60$ usec; left: $1.7 \mathrm{~V}, 130 \mathrm{~Hz}$, $60 \mu \mathrm{sec}$ ). The second patient regained the ability to engage in previously avoided social activities and benefitted from improved anger symptoms with unilateral stimulation (right: $1.2 \mathrm{~V}, 130 \mathrm{~Hz}, 60 \mu \mathrm{sec}$ ). Although initial results appear promising, published results of the completed study are necessary.

\section{TABLE 2. DBS trials}

\begin{tabular}{|c|c|c|c|c|c|}
\hline $\begin{array}{c}\text { Clinical } \\
\text { Trial }\end{array}$ & $\begin{array}{l}\text { Stimulation } \\
\text { Frequency }\end{array}$ & $\begin{array}{l}\text { Primary } \\
\text { Outcomes }\end{array}$ & $\begin{array}{l}\text { Secondary } \\
\text { Outcomes }\end{array}$ & $\begin{array}{c}\text { Anatomical } \\
\text { Target }\end{array}$ & Results \\
\hline NCT03416894 ${ }^{73}$ & NA & SAFTEE collateral effects scale & $\begin{array}{l}\text { HAM-D, BDI, BAI, CAPS, DTS, SPRINT, CGI, } \\
\text { GAF, SF-36, STAI, HAM-A, SDS, PANAS }\end{array}$ & $\begin{array}{l}\text { Subgenual } \\
\text { cingulate }\end{array}$ & NA \\
\hline NCT0209184372 & $130 \mathrm{~Hz}, 60 \mu \mathrm{sec}$ & Frequency \& severity of all adverse events & CAPS & BLA & Limited* \\
\hline
\end{tabular}

BAI = Beck Anxiety Inventory; BDI = Beck Depression Inventory; CAPS = Clinician-Administered PTSD Scale; CGI = Clinical Global Impression; DTS = Davidson Trauma Scale; GAF = Global Assessment of Functioning; HAM-A = Hamilton Anxiety Rating Scale; HAM-D = Hamilton Depression Rating Scale; NA = not available; PANAS = Positive and Negative Affect Schedule; SAFTEE = Systematic Assessment for Treatment Emergent Events; SDS = Sheehan Disability Scale; SF-36 = 36Item Short Form Health Survey; SPRINT = Short PTSD Rating Interview; STAI = State-Trait Anxiety Inventory.

* Published results of 2 patients (see text). ${ }^{59}$ 


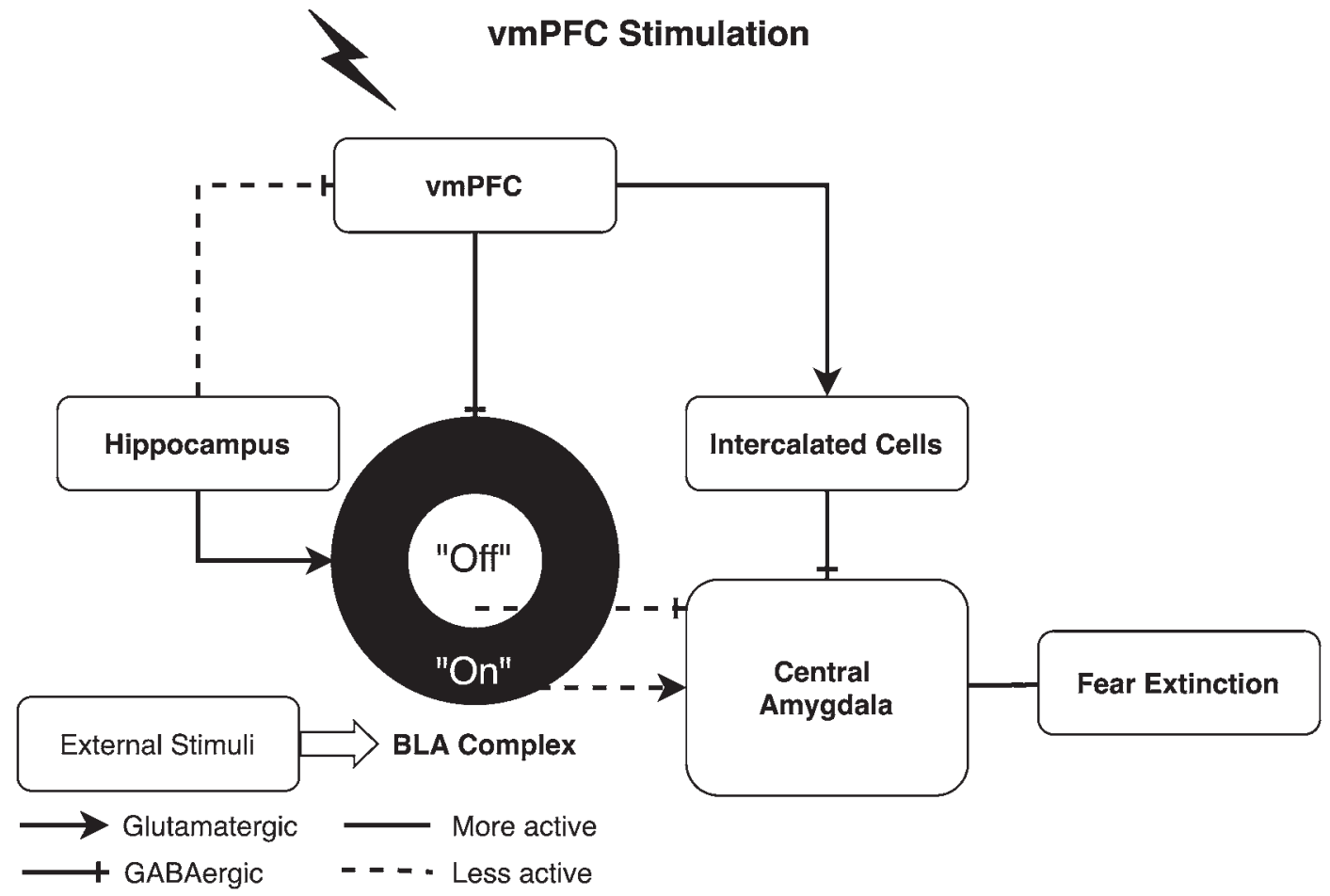

FIG. 2. Diagram representation of vmPFC stimulation for PTSD in a fear-persistent state. The concept underlying stimulation of vmPFC is its ability to reduce the activity of the central amygdala both via an overall increase in the GABAergic tone within the $B L A$ complex and via increased stimulation of intercalated cells in a fear-persistent state. High-frequency vmPFC stimulation may be able to mitigate hippocampal or an external stimulus's resultant stimulation of the central amygdala, thus biasing the network's activity toward fear extinction.

An alternative hypothesis for the use of DBS for treatment of PTSD involves changing the activity of regions distant from the target via activation of neuronal projections. ${ }^{22}$ Infralimbic neurons have dense glutamatergic projections to intercalated cells and are implicated in fear extinction and the prevention of $\mathrm{CE}$ cell activation via BLA neurons. ${ }^{28}$ Animal models have identified that highfrequency stimulation of the infralimbic neurons/vmPFC results in the decreased firing frequency of BLA principal cells, which is thought to be secondary to an increase in GABA release via interneurons, as well as increased intercalated cell stimulation and inhibition of CE. ${ }^{22,28}$ Reznikov et al. used mice with weak fear extinction, which may more closely resemble a clinical PTSD in humans. Stimulation resulted in mitigated fear responses, improved anxiety behavior, and prevented return of PTSD-like symptoms. ${ }^{22,60}$

Optogenetic studies have shown that inhibition of neurons projecting from the prelimbic cortex inhibits the expression of conditioned fear behaviors. In contrast, the inhibition of infralimbic neurons prevents the formation rather than retention of memories during fear extinction training. There is continued debate among comparative neuroanatomists on the exact homologies between the human and rodent PFC. Still, it is suggested that the vmPFC homolog in rodents consists of the prelimbic and infralimbic cortices, ${ }^{26}$ making the vmPFC an attractive potential target for the treatment of PTSD (Fig. 2). It is worth noting that findings from optogenetic studies may not directly transfer to DBS, because optogenetics allows cell type- specific alterations (but of course requires genetic alterations), whereas DBS is not specific at the level of cellular subtypes.

Closed-Loop Stimulation. The development of more refined neuromodulation protocols using closed-loop DBS, as opposed to open-loop DBS, could improve the precision of therapeutic stimulation. An open-loop system delivers only a preprogrammed stimulus. In contrast, a closed-loop system is capable of detecting and analyzing peripherally collected data, after which it is then able to deliver the appropriate stimulus to elicit the desired clinical outcome. Several central and peripheral biomarkers reflecting physical changes related to sympathetic activation such as alteration in heart rate or blood pressure, pupillary dilation, piloerection, skin conductive changes, or even endocrine markers related to a dysfunctional hypothalamic-pituitary-adrenal axis have been considered ${ }^{61}$ However, these biomarkers do not specificity represent the pathology associated with PTSD. The use of sensors in areas known to be related to the PTSD circuitry to measure local field potential changes in response to external stimuli may help improve specificity. In animal trials, increases in BLA potentials in the gamma range occurred after a trauma reminder. ${ }^{62}$ Currently there are 2 commercially available devices capable of closed-loop brain stimulation, both of which have research capabilities that allow for the extraction of data. ${ }^{61}$ Comparing peripheral and central biomarkers with local field potential recordings from both asymp- 
tomatic and symptomatic PTSD episodes could identify more reliable biomarkers.

The National Institute of Mental Health RDoC is a research platform that targets functional domains of psychiatric disorders in a multidimensional approach focused on behavioral and thought patterns, neurobiology, and genetics, intending to improve clinical outcomes. ${ }^{17}$ The RDoC approach will hopefully enhance researchers' ability to objectively measure symptoms across functional domains linked to known PTSD circuitry to specifically design behavioral tasks to assess the impact of neuromodulation within each domain. ${ }^{19}$ For example, rather than measuring symptom severity by using phenomenological symptom scales, which may be susceptible to subjectivity and bias, changes after treatment could be measured along dimensions of approach versus avoidance behavior or emotional stability versus lability that better quantify the underlying pathology. Recorded neuronal signals could then be combined with behavioral data and analyzed appropriately (e.g., machine or deep learning techniques) for a wide range of psychiatric conditions. ${ }^{19}$

Along these lines, the Defense Advanced Research Projects Agency initiated the Systems-Based Neurotechnology for Emerging Therapies (SUBNETS) program, a part of the US federal Brain Research through Advancing Innovative Neurotechnologies (BRAIN) Initiative, with the goal of creating implanted, next-generation, closedloop neural stimulators and therapeutic systems for the treatment of neuropsychiatric illnesses. ${ }^{63}$ Since its inception, there have been several publications that described the ability to predict changes in mood from intracranially recorded neural signals. Sani et al. developed dynamic neural encoding models to delineate timescales of decoded mood states in human subjects with epilepsy who self-reported mood intermittently over multiple days. ${ }^{64}$ Kirkby et al. discovered that increased variability between the amygdala and hippocampus correlated with variations in self-reported worsening mood and anxiety. ${ }^{65}$ Rao et al. demonstrated that unilateral stimulation of the lateral OFC led to acute improvements in mood among subjects with moderate to severe depression. ${ }^{66}$

These findings are an essential step toward the development of a closed-loop system for other psychiatric illnesses. Proposed designs would be able to record and stimulate multiple regions of the brain with onboard control and telemetry capabilities. ${ }^{19}$ Symptoms of PTSD are not continuous, and effective modulation of proposed targets may not require constant stimulation. Closed-loop systems could provide additional information regarding neural circuit dysfunction and improve symptom control in a more specific, "on-demand" manner.

\section{Conclusions}

PTSD is a widespread and often devastating psychiatric condition made up of a heterogeneous group of symptoms. In the most severe treatment-resistant cases, neurostimulation has garnered increasing interest. The multiple brain regions involved vary in their degree of contribution to the overall symptomatology, prompting consideration for treatment individualization. There is a need for improved techniques to identify therapeutic targets better. The "RDoC-style" transdiagnostic approach may provide much-needed objective data in assessing this psychiatric illness and its molecular biomarkers, improving our understanding of symptomatology and treatment response, and the likelihood of successful implementation of specific neuromodulatory strategies and closed-loop systems. We suggest research aimed at the use of pre- and postdeployment resting-state functional imaging among military members and high-frequency stimulation of the vmPFC as another potential target for those who develop TR-PTSD. Although there is hope that neuromodulation will become a viable treatment modality for PTSD, this concept remains theoretical, and further research should involve institutional review board-approved controlled prospective clinical studies.

\section{References}

1. Trimble MR. Post-traumatic stress disorder: history of a concept. In: Figley C, ed. Trauma and Its Wake: The Study and Treatment of Post-Traumatic Stress Disorder. Wiley; 1985: 5-14.

2. American Psychiatric Publishing. Diagnostic and Statistical Manual of Mental Disorders: DSM-5. 5th ed. American Psychiatric Publishing; 2013.

3. Laufer RS, Brett E, Gallops MS. Symptom patterns associated with posttraumatic stress disorder among Vietnam veterans exposed to war trauma. Am J Psychiatry. 1985;142(11): 1304-1311.

4. Dengler BA, Hawksworth SA, Berardo L, et al. Bilateral amygdala stimulation reduces avoidance behavior in a predator scent posttraumatic stress disorder model. Neurosurg Focus. 2018;45(2):E16.

5. Tanielian T, Jaycox L, eds. Invisible Wounds of War-Psychological and Cognitive Injuries, Their Consequences, and Services to Assist Recovery. Rand Corporation; 2008.

6. Koek RJ, Langevin J-P, Krahl SE, et al. Deep brain stimulation of the basolateral amygdala for treatment-refractory combat post-traumatic stress disorder (PTSD): study protocol for a pilot randomized controlled trial with blinded, staggered onset of stimulation. Trials. 2014;15:356.

7. Raskind MA, Peterson K, Williams T, et al. A trial of prazosin for combat trauma PTSD with nightmares in active-duty soldiers returned from Iraq and Afghanistan. Am J Psychiatry. 2013;170(9):1003-1010.

8. Bisson JI, Ehlers A, Matthews R, et al. Psychological treatments for chronic post-traumatic stress disorder. Systematic review and meta-analysis. Br J Psychiatry. 2007;190:97-104.

9. Brinker M, Westermeyer J, Thuras P, Canive J. Severity of combat-related posttraumatic stress disorder versus noncombat-related posttraumatic stress disorder: a community-based study in American Indian and Hispanic veterans. J Nerv Ment Dis. 2007;195(8):655-661.

10. Koek RJ, Schwartz HN, Scully S, et al. Treatment-refractory posttraumatic stress disorder (TRPTSD): a review and framework for the future. Prog Neuropsychopharmacol Biol Psychiatry. 2016;70:170-218.

11. Rona RJ, Jones M, Sundin J, et al. Predicting persistent posttraumatic stress disorder (PTSD) in UK military personnel who served in Iraq: a longitudinal study. J Psychiatr Res. 2012;46(9):1191-1198.

12. Solomon Z, Horesh D, Ein-Dor T, Ohry A. Predictors of PTSD trajectories following captivity: a 35-year longitudinal study. Psychiatry Res. 2012;199(3):188-194.

13. Morina N, Wicherts JM, Lobbrecht J, Priebe S. Remission from post-traumatic stress disorder in adults: a systematic 
review and meta-analysis of long term outcome studies. Clin Psychol Rev. 2014;34(3):249-255.

14. Eftekhari A, Ruzek JI, Crowley JJ, et al. Effectiveness of national implementation of prolonged exposure therapy in Veterans Affairs care. JAMA Psychiatry. 2013;70(9):949-955.

15. Panagioti M, Gooding PA, Tarrier N. A meta-analysis of the association between posttraumatic stress disorder and suicidality: the role of comorbid depression. Compr Psychiatry. 2012;53(7):915-930.

16. Johnson DR, Fontana A, Lubin H, et al. Long-term course of treatment-seeking Vietnam veterans with posttraumatic stress disorder: mortality, clinical condition, and life satisfaction. J Nerv Ment Dis. 2004;192(1):35-41.

17. Insel $\mathrm{T}$, Cuthbert $\mathrm{B}$, Garvey $\mathrm{M}$, et al. Research domain criteria (RDoC): toward a new classification framework for research on mental disorders. Am J Psychiatry. 2010;167(7): $748-751$.

18. Insel TR. The NIMH Research Domain Criteria (RDoC) Project: precision medicine for psychiatry. Am J Psychiatry. 2014;171(4):395-397.

19. Bari AA, Mikell CB, Abosch A, et al. Charting the road forward in psychiatric neurosurgery: Proceedings of the 2016 American Society for Stereotactic and Functional Neurosurgery Workshop on Neuromodulation for Psychiatric Disorders. J Neurol Neurosurg Psychiatry. 2018;89(8):886-896.

20. Gouveia FV, Gidyk DC, Giacobbe P, et al. Neuromodulation strategies in post-traumatic stress disorder: from preclinical models to clinical applications. Brain Sci. 2019;9(2):45.

21. Reznikov R, Binko M, Nobrega JN, Hamani C. Deep brain stimulation in animal models of fear, anxiety, and posttraumatic stress disorder. Neuropsychopharmacology. 2016; 41(12):2810-2817.

22. Reznikov R, Bambico FR, Diwan M, et al. Prefrontal cortex deep brain stimulation improves fear and anxiety-like behavior and reduces basolateral amygdala activity in a preclinical model of posttraumatic stress disorder. Neuropsychopharmacology. 2018;43(5):1099-1106.

23. Lissek S, Powers AS, McClure EB, et al. Classical fear conditioning in the anxiety disorders: a meta-analysis. Behav Res Ther. 2005;43(11):1391-1424.

24. Milad MR, Rauch SL, Pitman RK, Quirk GJ. Fear extinction in rats: implications for human brain imaging and anxiety disorders. Biol Psychol. 2006;73(1):61-71.

25. Besnard A, Sahay A. Adult hippocampal neurogenesis, fear generalization, and stress. Neuropsychopharmacology. 2016; 41(1):24-44.

26. Fenster RJ, Lebois LAM, Ressler KJ, Suh J. Brain circuit dysfunction in post-traumatic stress disorder: from mouse to man. Nat Rev Neurosci. 2018;19(9):535-551.

27. LeDoux J. The amygdala. Curr Biol. 2007;17(20):R868R874.

28. Duvarci S, Pare D. Amygdala microcircuits controlling learned fear. Neuron. 2014;82(5):966-980.

29. Admon R, Milad MR, Hendler T. A causal model of posttraumatic stress disorder: disentangling predisposed from acquired neural abnormalities. Trends Cogn Sci. 2013;17(7): 337-347.

30. Shin LM, Bush G, Milad MR, et al. Exaggerated activation of dorsal anterior cingulate cortex during cognitive interference: a monozygotic twin study of posttraumatic stress disorder. Am J Psychiatry. 2011;168(9):979-985.

31. Liberzon I, Sripada CS. The functional neuroanatomy of PTSD: a critical review. Prog Brain Res. 2008;167:151-169.

32. Blair RJR. Psychopathy, frustration, and reactive aggression: the role of ventromedial prefrontal cortex. Br J Psychol. 2010;101(pt 3):383-399.

33. Haden SC, Scarpa A. The noradrenergic system and its involvement in aggressive behaviors. Aggress Violent Behav. 2007;12(1):1-15.
34. Davidson RJ, Putnam KM, Larson CL. Dysfunction in the neural circuitry of emotion regulation-a possible prelude to violence. Science. 2000;289(5479):591-594.

35. Dileo JF, Brewer WJ, Hopwood M, et al. Olfactory identification dysfunction, aggression and impulsivity in war veterans with post-traumatic stress disorder. Psychol Med. 2008;38(4): 523-531.

36. Lanius RA, Vermetten E, Loewenstein RJ, et al. Emotion modulation in PTSD: clinical and neurobiological evidence for a dissociative subtype. Am J Psychiatry. 2010;167(6): 640-647.

37. Phelps EA, Delgado MR, Nearing KI, LeDoux JE. Extinction learning in humans: role of the amygdala and vmPFC. Neuron. 2004;43(6):897-905.

38. Wood JN, Grafman J. Human prefrontal cortex: processing and representational perspectives. Nat Rev Neurosci. 2003; 4(2):139-147.

39. Britton JC, Phan KL, Taylor SF, et al. Corticolimbic blood flow in posttraumatic stress disorder during script-driven imagery. Biol Psychiatry. 2005;57(8):832-840.

40. Diekhof EK, Geier K, Falkai P, Gruber O. Fear is only as deep as the mind allows: a coordinate-based meta-analysis of neuroimaging studies on the regulation of negative affect. Neuroimage. 2011;58(1):275-285.

41. Hopper JW, Frewen PA, van der Kolk BA, Lanius RA. Neural correlates of reexperiencing, avoidance, and dissociation in PTSD: symptom dimensions and emotion dysregulation in responses to script-driven trauma imagery. J Trauma Stress. 2007;20(5):713-725.

42. Craig AD. How do you feel? Interoception: the sense of the physiological condition of the body. Nat Rev Neurosci. 2002; 3(8):655-666.

43. Phan KL, Britton JC, Taylor SF, et al. Corticolimbic blood flow during nontraumatic emotional processing in posttraumatic stress disorder. Arch Gen Psychiatry. 2006;63(2): 184-192.

44. New AS, Fan J, Murrough JW, et al. A functional magnetic resonance imaging study of deliberate emotion regulation in resilience and posttraumatic stress disorder. Biol Psychiatry. 2009;66(7):656-664.

45. Der-Avakian A, Markou A. The neurobiology of anhedonia and other reward-related deficits. Trends Neurosci. 2012; 35(1):68-77.

46. Nawijn L, van Zuiden M, Frijling JL, et al. Reward functioning in PTSD: a systematic review exploring the mechanisms underlying anhedonia. Neurosci Biobehav Rev. 2015;51: 189-204.

47. Litz BT. Emotional numbing in combat-related post-traumatic stress disorder: a critical review and reformulation. Clin Psychol Rev. 1992;12(4):417-432.

48. Ferenczi EA, Zalocusky KA, Liston C, et al. Prefrontal cortical regulation of brainwide circuit dynamics and rewardrelated behavior. Science. 2016;351(6268):aac9698.

49. Falconer E, Bryant R, Felmingham KL, et al. The neural networks of inhibitory control in posttraumatic stress disorder. $J$ Psychiatry Neurosci. 2008;33(5):413-422.

50. Logue MW, van Rooij SJH, Dennis EL, et al. Smaller hippocampal volume in posttraumatic stress disorder: a multisite ENIGMA-PGC study: subcortical volumetry results from posttraumatic stress disorder consortia. Biol Psychiatry. 2018;83(3):244-253.

51. Aupperle RL, Melrose AJ, Stein MB, Paulus MP. Executive function and PTSD: disengaging from trauma. Neuropharmacology. 2012;62(2):686-694.

52. Maeda F, Keenan JP, Tormos JM, et al. Modulation of corticospinal excitability by repetitive transcranial magnetic stimulation. Clin Neurophysiol. 2000;111(5):800-805.

53. Rossi S, Hallett M, Rossini PM, Pascual-Leone A. Safety, ethical considerations, and application guidelines for the use 
of transcranial magnetic stimulation in clinical practice and research. Clin Neurophysiol. 2009;120(12):2008-2039.

54. Hemond CC, Fregni F. Transcranial magnetic stimulation in neurology: what we have learned from randomized controlled studies. Neuromodulation. 2007;10(4):333-344.

55. Trevizol AP, Barros MD, Silva PO, et al. Transcranial magnetic stimulation for posttraumatic stress disorder: an updated systematic review and meta-analysis. Trends Psychiatry Psychother. 2016;38(1):50-55.

56. Binder DK, Rau G, Starr PA. Hemorrhagic complications of microelectrode-guided deep brain stimulation. Stereotact Funct Neurosurg. 2003;80(1-4):28-31.

57. Sillay KA, Larson PS, Starr PA. Deep brain stimulator hardware-related infections: incidence and management in a large series. Neurosurgery. 2008;62(2):360-367.

58. Benabid AL, Benazzous A, Pollak P. Mechanisms of deep brain stimulation. Mov Disord. 2002;17(suppl 3):S73-S74.

59. Koek R, Langevin J, Krahl S, et al. Basolateral amygdala deep brain stimulation for treatment refractory combat PTSD: data from the first two cases. Brain Stimul. 2019; 12(2):429-430.

60. Bentefour Y, Bennis M, Garcia R, Ba-M'hamed S. Highfrequency stimulation of the infralimbic cortex, following behavioral suppression of PTSD-like symptoms, prevents symptom relapse in mice. Brain Stimul. 2018;11(4):913-920.

61. Bina RW, Langevin J-P. Closed loop deep brain stimulation for PTSD, addiction, and disorders of affective facial interpretation: review and discussion of potential biomarkers and stimulation paradigms. Front Neurosci. 2018;12:300.

62. Fenton GE, Spicer CH, Halliday DM, et al. Basolateral amygdala activity during the retrieval of associative learning under anesthesia. Neuroscience. 2013;233:146-156.

63. Emondi A: Systems-Based Neurotechnology for Emerging Therapies (SUBNETS). Accessed June 3, 2020. https://www. darpa.mil/program/systems-based-neurotechnology-foremerging-therapies

64. Sani OG, Yang Y, Lee MB, et al. Mood variations decoded from multi-site intracranial human brain activity. Nat Biotechnol. 2018;36(10):954-961.

65. Kirkby LA, Luongo FJ, Lee MB, et al. An amygdala-hippocampus subnetwork that encodes variation in human mood. Cell. 2018;175(6):1688-1700.e14.

66. Rao VR, Sellers KK, Wallace DL, et al. Direct electrical stimulation of lateral orbitofrontal cortex acutely improves mood in individuals with symptoms of depression. Curr Biol. 2018;28(24):3893-3902.e4.

67. Ahmadizadeh M-J, Rezaei M. Unilateral right and bilateral dorsolateral prefrontal cortex transcranial magnetic stimulation in treatment post-traumatic stress disorder: a randomized controlled study. Brain Res Bull. 2018;140:334-340.

68. Cohen H, Kaplan Z, Kotler M, et al. Repetitive transcranial magnetic stimulation of the right dorsolateral prefrontal cortex in posttraumatic stress disorder: a double-blind, placebocontrolled study. Am J Psychiatry. 2004;161(3):515-524.
69. Isserles M, Shalev AY, Roth Y, et al. Effectiveness of deep transcranial magnetic stimulation combined with a brief exposure procedure in post-traumatic stress disorder-a pilot study. Brain Stimul. 2013;6(3):377-383.

70. Kozel FA. Clinical repetitive transcranial magnetic stimulation for posttraumatic stress disorder, generalized anxiety disorder, and bipolar disorder. Psychiatr Clin North Am. 2018;41(3):433-446.

71. Kozel FA, Van Trees K, Larson V, et al. One hertz versus ten hertz repetitive TMS treatment of PTSD: a randomized clinical trial. Psychiatry Res. 2019;273:153-162.

72. Langevin J-P. Deep brain stimulation of the amygdala for combat post-traumatic stress disorder. U.S. National Library of Medicine. Accessed June 3, 2020. https://clinicaltrials.gov/ ct2/show/NCT02091843

73. Lipsman N. Deep brain stimulation for treatment refractory PTSD. U.S. National Library of Medicine. Accessed June 3, 2020. https://clinicaltrials.gov/ct2/show/NCT03416894

74. Osuch EA, Benson BE, Luckenbaugh DA, et al. Repetitive TMS combined with exposure therapy for PTSD: a preliminary study. J Anxiety Disord. 2009;23(1):54-59.

75. Watts BV, Landon B, Groft A, Young-Xu Y. A sham controlled study of repetitive transcranial magnetic stimulation for posttraumatic stress disorder. Brain Stimul. 2012;5(1): $38-43$.

\section{Disclosures}

Dr. Goodman received donated devices from Medtronic for an NIH research study. Dr. Sheth is a consultant for Abbott, Koh Young, and Neuropace. Dr. Storch is a consultant for Levo Therapeutics. He receives royalties from Wiley, Oxford, Elsevier, Lawrence Erlbaum, APA, and Springer. He receives honoraria from the International OCD Foundation. He received clinical or research support for the study described (includes equipment or material) from the NIH, the Texas Higher Education Coordinating Board, ReBuild Texas, the Red Cross, and the Greater Houston Community Foundation.

\section{Author Contributions}

Conception and design: Larkin, Viswanathan, Sheth. Acquisition of data: Larkin, McGinnis. Analysis and interpretation of data: Larkin, McGinnis, Snyder, Storch, Goodman. Drafting the article: Larkin, McGinnis, Snyder. Critically revising the article: all authors. Reviewed submitted version of manuscript: all authors. Approved the final version of the manuscript on behalf of all authors: Larkin. Study supervision: Larkin, Viswanathan, Sheth.

\section{Correspondence}

M. Benjamin Larkin: Baylor College of Medicine, Houston, TX. michael.larkin@bcm.edu. 